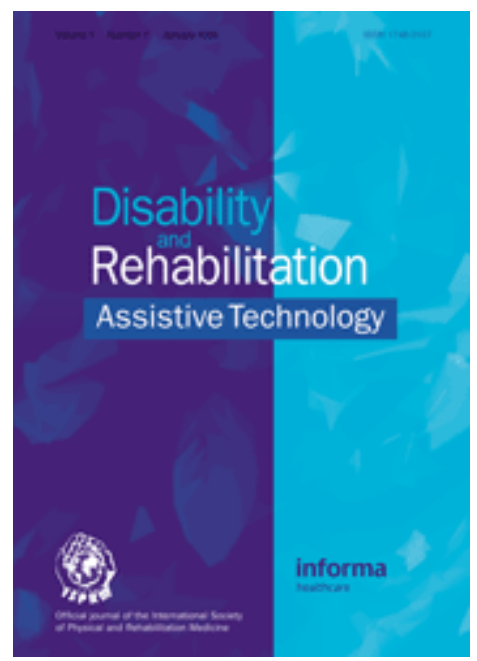

\title{
Understanding independent wheelchair transfers. Perspectives from stakeholders
}

\begin{tabular}{|r|l|}
\hline Journal: & Disability and Rehabilitation: Assistive Technology \\
\hline Manuscript ID & TIDT-08-2018-007.R1 \\
\hline Manuscript Type: & Original Research \\
\hline Keywords: & $\begin{array}{l}\text { wheelchiar users, occupational therapists, wheelchair transfers, } \\
\text { wheelchiar skills training, assistive technology }\end{array}$ \\
\hline \multicolumn{2}{|l}{} \\
\hline
\end{tabular}

\section{SCHOLARONE Manuscripts}




\section{Implications for Rehabilitation}

- The ability to transfer independently is extremely important for many wheelchair users and can affect not only their personal and social lives, but also the way they perceive themselves and are perceived by others.

- The use of a correct transferring technique plays an important role in reducing the effort required to complete a transfer and decreases the risk factors for both falls and upper limb injury development. Unfortunately, few wheelchair users have access to in-person training to develop transferring skills. The remaining people rely on a combination of peer observation, personal research and simple trial and error to figure out the movement strategies that better suit their needs.

- Currently available assistive technologies are perceived by wheelchair users as only partially successful in providing support during the execution of transfers. Transfer boards and other devices are often described as only useful for basic transfers and for individuals with reduced, but still sufficient, upper limb function. 
Table 1 Summary of participants' characteristics and their allocation between focus groups and interviews

\begin{tabular}{|c|c|c|c|c|}
\hline Participant & Gender & Medical condition & $\begin{array}{c}\text { Transferring } \\
\text { technique }\end{array}$ & Data collection method \\
\hline OT1 & F & NA & NA & Focus group \\
\hline OT2 & F & NA & NA & Focus group \\
\hline OT3 & F & NA & NA & Focus group \\
\hline OT4 & F & NA & NA & Focus group \\
\hline WU1 & F & Complex Regional Pain Syndrome & Sitting & Focus group \\
\hline WU2 & F & Spina Bifida & Sitting & Focus group \\
\hline WU3 & M & Spina Bifida & Standing & Focus group \\
\hline WU4 & F & SCI & Transfer board & Focus group \\
\hline WU5 & M & SCI & Sitting & Interview \\
\hline WU6 & M & MS & Standing & Interview \\
\hline WU7 & M & CP & Stitting & Interview \\
\hline WU8 & F & EDS & Transfer board & Interview \\
\hline WU9 & M & SCI & Sitting & Interview \\
\hline WU10 & M & SCI & Standing & Interview \\
\hline WU11 & F & MS & & \\
\hline
\end{tabular}




\section{Understanding independent wheelchair transfers. Perspectives from} stakeholders

Purpose: Transferring to and from the wheelchair is among the most important routine tasks for many wheelchair users-users. Transfers are also greatly affected by many personal and external factors. The purpose of this study is to investigate personal experiences, needs and concerns of wheelchair users in relation to wheelchair transfers performed in their everyday lives. Methods: Transfers are also greatly affected by many personal and external factors. However, the personal dimension of wheelchair users in relation to transferring tasks has never been explored. This article presents findings from a $\underline{A}$ series of focus groups and interviews were carried out with 11 wheelchair users and 4 occupational therapists to understand the perspectives, needs and concerns of wheelchair users. Data were analysed using a hybrid deductive and inductive approach of thematic analysis. Results: The seven themes identified emphasize the importance and complexity of wheelchair transfers. Transfers were described as gateways to independence that grant access to life opportunities, and community participation. FNonetheless, transferring skills are difficult to acquire and the concept of correct technique, although really important, is often poorly defined. Wheelchair transfers can be further complicated by the characteristics of the individual, the presence of upper limb pain, fear of falling or the characteristics of the environment. Despite the importance of transfer training to improve safety and reduce the risk of overload injuries, only a few people receive dedicated advice from health professionals. Currently available assistive technologies were perceived as only partially successful in providing support to wheelchair users during the execution of transfers, especially when environmental constraints make the transfer more challenging. Conclusion: Due to their multifactorial nature, creating effective solutions to improve any aspect of wheelchair transfers will require a collaborative effort from users, clinicians, designers and other professionals.

Keywords: wheelchair users; occupational therapists, wheelchair transfers, wheelchair skills training, assistive technology 


\section{Introduction}

Whenever we think about a wheelchair user we are likely to picture a person sitting in their wheelchair; perhaps pushing it, maybe even-of a Paralympian competing in an event. However, it is not often people consider how a person gets into and out of their wheelchair. This vital skill - transferring - enables independence for wheelchair users. Transferring between a car and their wheelchair was rated as the most important skill by both Paralympians and "regular" manual wheelchair users [1]; and transfers are essential for many daily activities such as getting out of bed, taking a shower, or using a toilet [2]. Wheelchair transfers are also physically demanding [3] and can potentially lead to falls [4] and upper limb injuries [5]. Injuries such as carpal tunnel syndrome and rotator cuff tears cause wrist and shoulder pain. The subsequent pain reduces the person's ability to transfer and to propel their wheelchair, which leads to an overall reduction of the quality of life [6]

The number of times a person transfers to and from the wheelchair in a single day, the type of transfers performed and the movement strategies adopted to carry out the transfer vary greatly across different individuals $[7,8]$. These differences depend not only on the impairment and the environmental conditions, but also on habits, lifestyle and personal preferences. Several studies have focused on quantifying the kinematics and kinetics aspects of wheelchair transfers [9,10], while others have assessed the quality and safety of wheelchair transfers using a standardized clinical scale $[11,12]$. The study which has taken the most comprehensive approach to understanding the issues surrounding wheelchair transfers and suggesting potentially new directions for future research was carried out by [13], who conducted a series of focus groups with a cohort of 31 experts. Results highlighted the need for further investigation into the relationship between internal (pain, fear, spasticity, confidence) and external (gaps, poor wheelchair design, barriers) factors affecting the performance of wheelchair transfers, 
and the unique dimension of these relationships for different individuals. However, this study focused mainly on the opinions of professionals physiotherapists and researchers) rather thanand wheelchair users. and although all participants had considerable experience in wheelchair related fields, most were professionals rather than wheelchair users.Clinicians, researchers or designers with sufficient expertise might be able to sympathize and even understand the experiences of many wheelchair users, but they lack their personal perspectives [14].

In many respects wheelchair transfers represent a gateway for independence for wheelchair users. Transfers not only have a huge impact on the functional life of a wheelchair user but they can also shape internal and external perception of the individual and their perceived disability [15]. Despite this, the personal dimension of wheelchair users in relation to transferring tasks has never been explored.

The qualitative exploration of disability related issues ${ }_{2}$ and the implications of their findings for the design of assistive technologies or the formulation of clinical interventions represents a growing area of interest. The interview study carried out by [16], for example, -highlights how the value amputees attributed to upper limb prosthetics goes far beyond their functional impact. Prosthetic devices become an integral part of people's identity and their impact on the presentation of self is equally, if not more, important than their "assistive" function. Similarly, participants in the study carried out by [17] preferred unobtrusive inputs and outputs in the interfaces for mobile computing devices ${ }_{2}$ as they did not wish to add devices that will change the form factor of their power wheelchairs. This is not surprising, as many wheelchair users "wear their wheels" in the same way that amputees wear their prosthesis [18].

In order to gain a comprehensive understanding of the personal perspectives, needs and concerns of wheelchair users in relation to everyday transferring activities 
direct consultation is essential. Furthermore, the insights captured from such an investigation could complement the quantitative data collected from laboratory and field studies, and provide more concrete design and clinical insights that ${ }_{2}$ in turn, could help to shape novel support strategies aimed at facilitating the performance of wheelchair transfers in people's everyday lives. Based on these considerations, the aim of this study was to collect direct testimony, from both wheelchair users and occupational therapists (OTs), aboutef the most important and difficult aspects of independent wheelchair transfers. In comparison to the main body of literature related to wheelchair transfers, this study moves beyond the evaluation of objective factors such as the impact of impairment, technique and environmental barriers or facilitators, and encompasses the personal elements that shape the individual experiences of wheelchair users.

\section{Methods}

\section{Participants}

To get a more comprehensive and objective overview of difficulties encountered during wheelchair transfer performance participants were recruited among both OTs and wheelchair users. Participants were recruited from a laboratory database and through Disabled People Organizations. Inclusion criteria for the study were: aged over 18 years, and having at least six months of experience as a wheelchair user or as an OT with specific competencies in wheelchair skills training. Additionally, wheelchair users had to be able to perform independent transfers using a standing or sitting technique, with or without a transfer board. The study was approved by the (Removed for Anonymization) Ethics Committee and all participants were asked to sign an informed consent form before taking part in any activity. A total of 15 people, $\underline{4}$ four OTs and 11eleven wheelchair users who satisfied the inclusion criteria were recruited for this 
study. The four OTs had been working in a Spinal Cord Injury (SCI) rehabilitation centre for a period ranging from 9 months to 16 years. All wheelchair users had at least one year of experience using their wheelchair as a primary means of mobility. Five wheelchair users reported using a standing technique for most of their transfers, $\underline{4}$ four used an unassisted sitting technique and 2 two benefitted from the use of a transfer board for most of their transfers.

\section{Materials and Procedure}

Due to the inherent difficulties encountered when recruiting wheelchair users, the qualitative study was carried out using a mixture of focus groups and semi structured interviews. Distribution of participants between focus groups and semi-structured interviews alongside participants' gender and the transferring technique reported by the wheelchair users are shown in Table 1. According to their availability, 9 nine participants were enrolled in two focus groups ( 5 wheelchair users and 4 OTs), whereas the remaining $\underline{6}$ six wheelchair users took part in semi structured interviews. Wheelchair users and OTs were assigned to different focus groups. This separation was planned in order to create more homogeneous groups and facilitate sharing of opinions without the interference of unbalanced power dynamics.

\section{$\underline{\text { Table } 1}$}

For consistency reasons, the questioning route was the same for both focus groups and interviews. Questions asked in both focus groups and interviews were aimed at exploring various aspects of wheelchair transfers and ranged from questions concerning transfer technique, $\underline{\text { to}}$ and the difficulties encountered when learning transferring skills. They also explored how, when transferring on a daily basis, wheelchair users dealt with pain, fear of falling and their thoughts on current and potential assistive technologies for transfers. Furthermore, to capture additional details concerning the personal dimension 
of wheelchair transfers a simple cultural probing technique was used [19]. Cultural probes are a common design technique in which participants use artefacts, in this case $\underline{\text { their mobile phone or a simple diary, to record specific events that would be difficult for }}$ the researcher to observe in their natural context [19]. Before the start of the interview or the focus groups $s_{2}$ all wheelchair users were asked to present a short video or write down and read a description of a transfer that they considered particularly important. Thematic analysis was chosen as the analysis method for this study due to its rigor, flexibility and usefulness in summarizing relevant features of complex datasets [20]. Focus groups and interviews were audio recorded and transcribed by the first author. Transcripts were then analysed and coded using a hybrid deductive, where themes are established a priori according to the coding framework established by the researcher, and inductive, where no predetermined framework is used and the themes are built in a data-driven way, approach as suggested by [21]. Coding was carried out in an iterative fashion until saturation was reached [22]. Four key themes were established a priori based on the aim of the study and the questions asked in the interviews were: "Important elements of wheelchair transfers' performance", "Difficulties when transferring”, “Transfers and pain”, “Transfers and ATs". Transcripts were initially revised individually by the first author and significant parts relevant to these four themes were highlighted and annotated to create content labels and descriptive codes. As the analysis progressed, codes were compared, renamed, split and combined to create the most comprehensive and coherent description of the data; this was conducted by both authors. Similarly, codes were grouped into relevant themes in an iterative fashion. Themes were first defined as they emerged and subsequently reviewed, refined and renamed in a iterative fashion to ensure the correct interpretation of the data [20]. A second inductive analysis was conducted to identify other relevant codes that 
emerged from the data collected. After comparative examination carried out between the two authors and triangulation with pre-identified codes and themes [23], three additional themes were extracted from the data.

\section{Results}

Seven main themes were identified from the analysis of all transcripts. Six themes were considered highly relevant to the scope of the current study. Although the seventh theme - 'Wheelchair users not wheelchair bound' - might appear not directly in line with the aim of our research, -it remains included. This is because several wheelchair users highlighted the issue as being extremely important ${ }_{2}$ and one that directly influences wheelchair transfers. Despite the different backgrounds and experiences of the participants, all themes consistently emerged and recurred across focus groups and interviews.

\section{The value of wheelchair transfers (1)}

Both OTs and wheelchair users clearly attributed extreme importance to the ability to transfer in and out of the wheelchair. OTs underlined how transfers represent a gateway to independence from the beginning of the rehabilitative process for people with SCI. Newly injured people initially find themselves in a bed and the very first step towards independence is learning the skill to be able to get out of the bed, and into a wheelchair. Therefore being able to independently transfer from the bed to the wheelchair allows people to reach their first level of independent mobility. Successive transfers to and from toilets, car seats or couches open up new possibilities for mobility, self-care, entertainment and interaction with others. Similarly, the videos and comments shared by wheelchair users, regardless of impairment, years of wheelchair use and transferring 
technique, showed how the ability to transfer in and out of the chair was crucial to living life.

Emphasis was put on the value of transfers in the context of: family life, work, ability to travel and mobility in general.

WU5 "I get on the couch in the play room to read and play with my little boy" WU11 "I transfer onto a perching stool when I teach to my student because when I was teaching from my wheelchair my students couldn't see me or hear me and this was starting to affect my work"

WU4 "I drive everywhere, I am in and out of the car constantly, so car transfers are very important to me"

WU7 "With muscles, if you snooze you lose. If I didn't transfer it would be far too easy for me not to exercise".

\section{The four pillars of wheelchair transfers (2)}

When describing important factors in the performance of wheelchair transfers, four elements were repeatedly emphasised by both wheelchair users and OTs: technique, confidence, balance and strength. Having sufficient strength, in upper or, lower limbs, or both, is a necessary requirement for both sitting and standing transfers. Depending on the environmental conditions, certain types of transfers, such as car transfers, might require a greater amount of strength. This results in individuals needing to practice more before being able to safely attempt them.

\footnotetext{
WU9 "I was told from the beginning that I would have been able to do a car transfer, but they needed me to get stronger first. I had to wait five months before they let me try".
}

Insufficient strength can also affect the transferring technique and is a motivating factor in the decision of a person to use a transfer board. Additionally, strength affects the individual's ability to cope with both horizontal and vertical gaps. As expected, upper 
limb strength was described as a necessary requirement for sitting transfers, however it was also reported to be a great asset for individuals who usually perform standing transfers, particularly in difficult situations.

WU1 "When my legs are not really behaving, I have to hold on to something and pull myself up. Otherwise I can't do it”.

Although strength is important, another element playing a determinant role is the use of a correct transferring technique. Both wheelchair users and OTs agreed that the most crucial element of a good transferring technique is a sufficient forward lean of the trunk, which creates momentum to relieve weight from the buttocks and helps to guide the body in the correct direction.

OT1 "For most people the core it's really the technique, and if you do the technique properly most of the time you won't have any problem".

The degree of forward lean was also described as being correlated with the gaps separating the starting and target surfaces. However, achieving a sufficient forward lean was considered the most difficult skill to achieve, particularly for people with SCI due to their lack of trunk control.

WU4 "The lean is very important. The bigger the gap you have to cross the more you have to lean. The less control of your muscles you have, the harder it gets because you just end up on your face".

On the other hand, the concept of correct technique seemed both less defined and less important for individuals performing standing transfers. In this population, the description of transfers' strategy was notably less standardized. Furthermore, maintaining correct balance was given higher priority compared to implementing strategies to reduce the load on upper and lower limbs. For this reason, several 
wheelchair users preferred to reduce the degree of forward lean, andinstead reliedy on their arms to push themselves up. Additionally, people often used external supports, such as handles, rails, walls or furniture to maintain balance once standing position is reached

WU6 "When I transfer I need to have armrests on the wheelchair to rise from and then I often need something to grab onto when I stand up"

Regardless of impairment and transferring strategy, one element that affected how people transfer in and out of the wheelchair is confidence. Lack of confidence in technique, strength or balance can lead people to perform transfers incorrectly. When feeling insecure some people tend to transfer too fast to "get over and done with it" (OT3), which can lead to falls or injuries. Others might have hesitations that limit their movements, making and make the transfer more difficult due to a more backward position of the center of mass and subsequent lack of momentum. The individual's level of confidence can be affected by several factors from a reduction of their physical capabilities due to fatigue or,-to a challenging environment. Additional training, practice and the presence of a spotter to supervise the transfer were suggested as potentially helpful measures by many of the OTs and wheelchair users.

\section{Internal and external difficulties (3)}

There are several elements that can make transferring harder for wheelchair users; these can be divided into internal and external difficulties. Internal difficulties are often

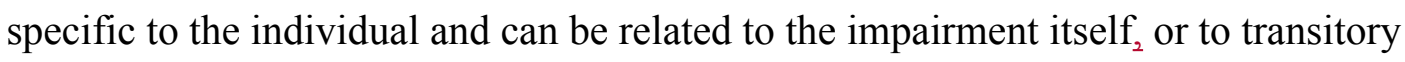
circumstances such as tiredness. Spasticity (abnormal and uncontrolled movement of a body part) was reported to be the main internal difficulty for many wheelchair users performing sitting transfers. 
WU1 "It's hard enough to transfer if your legs aren't working, it's harder if they are kicking".

On the other hand, people performing standing transfers often have balance issues, which are particularly severe when getting up for a transfer due to the change of position. Several wheelchair users also pointed out how fluctuations in their impairment, due to tiredness or their medical condition, can pose extreme challenges, forcing them to constantly evaluate and change their strategies based on their available capabilities.
WU8 "The symptoms I have change a lot, it's the nature of my condition. Some days I can walk a bit, while if I am having a really bad day, I am just crawling and I struggle to even get up... It's just hard to know it before you try something stupid..."

External difficulties were more varied and often described in association with a particular type of transfer. Large gaps in height and width were one of the most commonly reported difficulties, alongside lack of space to correctly position the wheelchair, inefficiency of wheel locksehair brakes, instability of transferring surfaces and the degree of bucket of the seat (created when the seat is higher towards the knee compared to the hips). Wheelchair users who regularly performed transfers to and from sport wheelchairs complained about the absence of wheel locks, breaks which poses significant challenges and increases the danger of these transfers. Participants who performed standing transfers complained about the current design of wheelchair footplates, as they can be difficult to move and can prevent the individuals from placing their feet appropriately when transferring.

WU11 'Because my balance is poor I can't bend forward to flip the footplates up, but then they are in my way. I can't step over them because they are too far away and I can't step to the side of them". 
Participants also mentioned the importance of several circumstantial factors. Rain, sweat or being wet after a shower can make sliding along a transfer board considerably more difficult. One difficulty that was specifically associated with car transfers was the potential presence of uneven or sloped terrain where the car is parked. Individuals performing standing transfers were particularly concerned about the potential negative effect this has on their balance; whereas people performing seated transfers described the difficulty of transferring while having to make sure that their wheelchair didn't accidentally move. Lastly, one difficulty reported often by both wheelchair users and OTs was related to the challenge of dealing with unfamiliar situations and generally awkward transfer set ups. These challenges are common when travelling on planes, staying in hotel rooms or visiting friend's houses and they force people-need to adapt their technique and develop workarounds with little or no possibility to prepare in advance.

\section{Learning how to transfer (4)}

Although most participants highlighted the importance of using a safe and efficient transferring technique, we discovered that only a-four wheelchair users had received appropriate training to learn how to transfer. Individuals with SCI were the only people who received formal training and guidance from medical professionals on how to develop safe transferring skills. Among other participants, some reported having learned mainly from peer observation.

WU2 "Everything I have learned, I have learned from watching other people around me, modify it and then do it myself".

When direct observation was not possible, virtual resources were used in the attempt to fill the gap. 
WU3 "I ended up googling and going to YouTube to watch videos of transfers".

Other participants relied solely on their own experience and learned through a long trial and error process.

WU1 "Everything I have learned, I have learned from falling and not doing it again".

Participants did not seem to think that their lack of training had affected their capabilities. However, some participants, such as WU8 expressed concerns about the potential damage that could result from their absence of training.

"If you don't learn to do stuff properly the chances are that before or after I am going to do myself an injury because of bad technique in my own wheelchair..."

Even when transfer training was provided, it was often in controlled clinical settings where barriers were reduced to a minimum, and assistance could be provided upon request. Some people declared that they learned all the skills they needed during rehabilitation. Other participants noted how, despite judging the training they received in the hospital extremely useful, their transfer strategy needed to be modified in order to be independent and safe once they went back home.

WU4 “After discharge, you can't stick to 'by the book' transfers that you were taught. You have to try and see what is going to work for you".

\section{The negative consequences of transfers Fear the falls but bear the pain (5)}

Avoiding falls when performing wheelchair transfers was found to be one of the primary concerns for the majority of participants. The specific reason behind this fears varied across different individuals. Some people were apprehensive about the potential injuries that a fall could result in, while others were more worried about the potential 
reaction of people who witnessed the event.

WU8 "For me a lot of the stress around falling is about how people are going to respond. A lot of time people try to help you, but they only pull you around and make everything harder and it's hard for you to direct them properly".

Fear of falling wasn't necessarily seen as a negative thing as it prompts wheelchair users to take care of their technique and avoid unnecessary risks. On the other hand, excessive fear was reported to have a negative effect on both confidence and transfer technique. Notably, two participants seemed to have little or no concern about the possibility of incurring a fall when transferring. For WU1 falls were reported so frequently that they were considered a common occurrence. While WU7 stated that fear was pointless as transfers were an integral part of his daily routine.

"I don't have a choice about transferring because of the impact it has on my quality of life. And, if I fell, I would have to get back on the bike anyway... So I am not scared".

Pain in the upper or lower limbs was also commonly associated with wheelchair transfer performance. Some participants described how pain necessitated changes tocaused them to change the way in which certain transfers are performed.

WU2 "When my carpal tunnel is very bad I need to push on my forearms rather than my hands to get out of the bath".

In other instances people found a way to make the process of transferring easier.

WU10 "When I get out of the bath I usually take the plug away and push myself up. When my shoulder was very sore I started to leave the plug in so I could get the maximum assistance for my lift".

Other wheelchair users reported attempting to reduce the frequency of transfers, 
whenever possible. However, when transfers were considered important for their daily routine they were performed regardless of the pain.

WU4 "If I can I just try to do less, don't hop on the couch or in and out of the car too much. I try to limit but I still have to do those transfers".

For some participants, pain was simply part of their transfers and, when bearing the pain was the only alternative to risking a fall, it was considered a preferable alternative.

WU3 "My shoulder just screams at me every time I load too much and this happens with every transfer. But if I try to do it in another way I will just fall over so I have to deal with it".

\section{Perspectives on assistive technology (6)}

The only assistive technologies (ATs) to facilitate wheelchair transfers that all participants were familiar with were transfer boards. The majority of participants reported using a bath board to transfer into the bath and two participants (WU4 and WU9) used transfer boards for all their transfers. Opinions on transfer boards were generally positive ${ }_{2}$ as they were considered functional objects which were fit for purpose. However, transfer boards were commonly described as helpful only for level transfers or transfers were the height gap was moderately low. Transfers with considerable height gaps between the two surfaces resulted in the board having an excessive slope causing the person to slide down in the middle of the transfer. Additionally, several participants complained about their weight, the difficulty of carrying them around and the fact that they can be easily damaged. OTs also noted how, for some wheelchair userWUs, particularly those with reduced upper limb function, picking up and positioning the transfer board correctly could represent a challenge in itself. WU10 expressed concerns about using ATs unless they were absolutely necessary as he felt they could, over time, decrease his ability 


\begin{abstract}
"The available equipment is good, but you don't want to start to use assistive technologies too soon because you need to keep your strength and ability as high as possible. Once you start using a transfer board you are not going back because you are not doing any training or exercise that will help you do a clean transfer".
\end{abstract}

Both wheelchair users and OTs expressed a desire for improved transfer boards or other new ATs that could support people when transferring in and out of their wheelchair. Some suggestions concerned wheelchair modifications such as retractable footplates that would not get in the way, or extendable armrests that could offer support when the person is standing up to transfer. Others focused more on improved versions of transfer boards. Some suggestions were for low tech solutions such as telescopic or collapsible transfer boards that could be easily carried around and function as a portable tray when needed. Alternative ideas involved a more complex board that could deal with greater height gaps, such as a transfer board with steps or transfer boards with an embedded sliding seat that could lock in place allowing the user to break the transfer into smaller motions. One participant advocated for a motorized transfer board featuring a conveyor belt mechanism that could safely carry wheelchair users with more limited mobility.

Ideas were not only related to transfer boards. Other suggestions included a device that could be used to level all transfers by raising the height of the lower surface, a system that could keep the shoulders in a stable and safe position during transfers or a glove that could be used to increase the grip when relying on slippery supports during transfers.

Finally, several participants mentioned the need for resources, not necessarily ATs, which could help people learn how to transfer by providing suggestions, guidelines and tips for safe and efficient technique regardless of the medical condition of the individual. Suggested solutions varied from a digital repository of information to chest placed sensors able to provide real-time bio-feedback on the optimal trunk 
forward lean for wheelchair users with SCI. Regardless of their format, instructions and guidance would need to be flexible enough to be adaptable to different individuals, and span across various situations. It was also emphasised that information concerning correct transferring technique should always include visual material as a simple verbal explanation was considered insufficient.

WU7 "You have to be able to show it, not just explaining it. If I don't see it I am never going to get it".

\section{Wheelchair users not wheelchair bound (7)}

The last theme that emerged from the analysis was not directly in line with the aim of the current study. However, it brings attention to an important issue that impacts wheelchair users and that can also directly affect wheelchair transfers. All participants, whose primary medical condition was not SCI, described episodes highlighting how both the public perception and the net of services related to wheelchair provision, training and assistance are shaped around the stereotype that sees all wheelchair users as individuals with a SCI. An example of this discrepancy was given by the fact that, among participants of the current study, no individual with an impairment different from SCI had received any training on transferring technique. Even when people researched for information on their own, they were unable to find guidelines or advice from health professionals that could guide their learning.

WU3 “I didn't really get any wheelchair skills training or transfer training... I remember I tried to look online as well ages ago but I couldn't find anything useful for me".

Several wheelchair users also mentioned how they often feel the pressure of this stereotype when in public settings. They explained how this can directly affect their behaviorbehaviour as they seek to avoid potential judgement from strangers 


\begin{abstract}
WU11 "My condition is not constant. The thing that annoys me is that a lot of the times I could get up and take a few steps, but often I don't do it. It's like I always have to behave as if it was a bad day for me. Which is actually the worst thing I can do for my own health. But people don't react well if they see you on the chair one day and walking the next day".
\end{abstract}

However, WU7 also pointed out how transfers have an intrinsic value in their ability to challenge stereotypes associated with wheelchair users, by presenting a separate image of the person and the chair.
"When I transfer onto a chair in a restaurant I can see the shock on the waiters face and I can understand them. If you ask anybody to picture a wheelchair user they will picture him [or her] on a chair, and I think there is something quite disruptive about moving away from the chair. It can help change people's perception".

\title{
Discussion
}

The main goal of the current study was to gain a deeper understanding of the experiences of wheelchair users in relation to transferring tasks. The diversity of impairments, transferring techniques and lifestyles among wheelchair users and OTs allowed us to capture a variety of viewpoints and ensured variability among individuals' opinions. Surprisingly, despite the participants' diversity, the seven themes identified were present in all focus groups and interviews. However, the relevance of different elements varied depending on the individual's characteristics. For example, the importance of training and technique was highlighted several times by OTs, individuals with SCI and subjects who acquired a disability in their adulthood and who had received training. On the other hand, participants who were born with a medical condition which required the use of a wheelchair for mobility, attributed considerably less importance to the concept of proper technique and standardized training. This can be explained not only because of the lack of training received, but also because learning 
to transfer is simply a part of normal skill acquiring process of development for a person - people have learnt to transfer in the same way people who walk have learnt to walk.

Differences were also found between participants of different age. Older wheelchair users were more concerned about the possibility of falling while performing a transfers, whereasile younger participants were less worried about their safety.

Interestingly, all wheelchair users who participated in the study made a point of highlighting, not only how important their transferring skills are for their independence, but also how overlooked they usually are by clinicians, other professionals and often themselves. Several people reported how it was difficult to describe their transfer technique in detail ${ }_{2}$ or the difficulties they might encounter when transferring; one reason given was that questions abquestions were not posed in a way to ascertain this information-out wheelchair transfers were rarely asked during standard clinical interactions. People appreciated being asked about wheelchair transfers. The focus group gave participants an opportunity to learn from each other and share the personal strategies they use to facilitate transfers. This process highlighted how rarely people exchange ideas and expertise on transfers. A potential explanation for this lack of information exchange among peers might be related to the fact that transfers belong to the personal sphere of the individual. Transfers often take place within the house or when the person is alone, which might reduce opportunities for observation or exchange of information. More complex transfers into and out of a bath for example. In contrast, it is easier to observe 'other' wheelchair users outdoors and pushing wheelchairs in challenging environments, such as propulsion on steep slopes or rough terrain. This might make it easier to observe strategies that other users employ or ask for suggestions on how to deal with specific difficulties. Additionally, the importance of transferring skills and the potential loss of independence that can arise from the inability to transfer 
without assistance can lead some users to become overly sensitive of others people's feelings and consequentially transfer less often around other wheelchair users.

\section{WU7 'Sometimes we don't want to boast about it because some people can't do it. It's like an unspoken politeness..."}

Overall, the seven themes identified in this study emphasise the complexity of wheelchair transfers. Transferring skills are difficult to acquire and the concept of correct technique, although extremely important, is often poorly defined. As reported by [13], wheelchair transfers can be further complicated by the built environment or the design of the wheelchair. Additionally, internal factors such as pain, spasticity, tiredness and fluctuations of the individual impairment can make the task even more difficult. However, due to their importance for the person's independence and quality of life, transfers are often seen as unavoidable.

-Due to their complexity and variability, learning how to transfer can be a challenging task for wheelchair user. Unfortunately, only people with SCI tend to receive formal training from medical professionals while other wheelchair users are often left to figure things out for themselves.

Unsurprisingly, mostall participants highlighted the importance of training in order to develop effective transferring skills and some participants expressed a concern that their lack of training could negatively affect their safety and increase the likelihood of developing injuries overtime. Previous studies have also shown that appropriate training can successfully improve safety and effectiveness of transfers and other wheelchair $\underline{\text { skills, particularly if delivered to new wheelchair users [24]. Additionally, improving }}$ transferring technique can help reduce the force required to perform the transfer [25]. In-person transfer training appears to be inaccessible for many wheelchair users and, 
when provided, might not be sufficient to provide wheelchair users with effective skills to perform real world transfers $[\underline{124,25,26,27] . ~ T h e ~ u s e ~ o f ~ a ~ w e b-b a s e d ~ t r a n s f e r ~ t r a i n i n g ~}$ module has been shown to significantly improve the transferring technique of wheelchair users. Technique gains tested immediately after training and after a short follow-up period were comparable to the one obtained with in-person training [28 26$]$. OThe availability of enline resources caneuld help provide wheelchair users with much needed training material that will allow them to develop transfer skills when in-person training is not possible and online videos can be . Additionally, web-based open source material could be consulted on-demand when people are faced with new and challenging situations. Interestingly, online material for wheelchair skills training developed by researchers and clinicians is available from several websites (e.g. www.whizz-kidz.org.uk, www.wheelchairskillsprogram.ca). However, participants seemed unaware of these resources, which potentially suggests that the channels used to diffuse these information fail to reach the target audience.

AT for transfers, and in particular transfer boards, generally received positive feedback. Nonetheless, they were found to be of limited use when the circumstances became more challenging or the users were more skilled. This is interesting as it highlights the fact that current ATs seem to be designed to facilitate people with basic tasks, but their ability to "bridge the gap" is often insufficient for more challenging situations. Additionally, transfer boards, or other ATs for transfers, seemed to be targeted onlytowards people with reduced upper limb function. This makes them inadequate, or counterproductive in enhancing more skilled individuals. Part of the reason for this can be linked to the fact that in assistive technology $\underline{\mathrm{AT}}$ design there is a tension between the need to meet the specific needs of a disabled individual and the need to develop a product with the largest market base possible in order to generate 
sufficient profit [2927]. This can lead to the tendency to develop a product based on a persona with average capabilities for use in an average or standardized context.

Challenging situations and particularly skilled wheelchair users therefore, fall outside of the target audience of the product, making mainstream assistive technologies $\underline{\mathrm{ATS}}$ unsuitable for use. Engaging wheelchair users, and people with disabilities more generally, in targeted co-design projects, would help design for people beyond a fictional 'normal', who due to the nature and context of the activity or their capabilities, find themselves at the edges of the Gaussian curve [3028].

Finally, results from the current study bring further attention to an issue that has recently been mentioned by several researchers. Experts who participated in the Independent Transfer Workshop [3129] identified the need to include in future research studies more wheelchair users without SCI in order to improve generalizability of findings. The tendency to use the SCI group stereotype to represent wheelchair

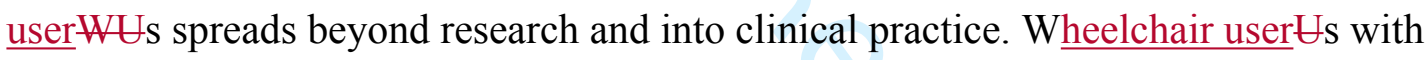
impairments other than SCI had received little or no training on transferring technique and wheelchair skills. Furthermore, this population are often unaware of the existence of ATs that might improve their abilities and can struggle to get assistance and funding for basic equipment from a health system that seems unable to deal with them because they "do not fit in the guidelines". The weight of this stereotype goes beyond the medical community and shapes public perception so strongly it causes wheelchair userWUs to modify their behavior based on the expectations of others, like the actors on the stage described by [3230].

\section{$\underline{\text { Limitations }}$}

Although this study shade light on some new and interesting personal perspectives of 
$\underline{\text { wheelchair users in regard of transferring activities, it is not free from limitations. }}$ Firstly, the small sample size suggests caution in the interpretation of the results. $\underline{\text { Secondly, all the OTs who took part in the study had worked mainly in SCI }}$ $\underline{\text { rehabilitation. SCI rehabilitation centres are highly specialized institutions dedicated to }}$ patients with one particular condition. The experience of the OTs interviewed in the $\underline{\text { study might be different from others who work in different contexts and are in contact }}$ with patients who different kinds of impairments. Thirdly, in order to accommodate the needs of participants and increase chances of recruitment, a mixture of focus groups and interviews where used for data collection. Although the use of heterogeneous data collection methods in not unheard of in qualitative studies, the different data collection contexts might have affected participant responses and this suggests when generalising the results.

\section{Conclusion}

Biomechanies studies and clinical observations can help to identify and quantify some of the challenges and risks that people encounter when transferring to and from their wheelchairs. However, these studies can only give a general glimpse of wheelchair users' experiences in relation to transferring activities. To this endIn this study, a series of focus groups and semi structured interviews with both wheelchair users and OTs were conducted with the aim of gaining a deeper understanding of the perspectives, needs and concerns that wheelchair users encounter when transferring in their everyday lives, not only due to the built environment or their disability, but also in relation to their lifestyle and personal experiences.

The seven themes identified through this qualitative investigation-highlight the importance of wheelchair transfers for in relation to both the personal image and the quality of life on the individual. Transfers were described as gateways to independence 
which could progressively unlock new levels of personal mobility for the persen, granting new opportunities for participation in life. However, their value comes at a cost as transfers wereare often described as difficult, painful and sometimes risky.

Challenges encountered during transfers can be linked to a combination of personal and environmental factors. Due to their multifactorial nature, creating effective solutions to improve any aspect of wheelchair transfers from technique, training, public perception, accessibility or assistive technologiesATs will require a collaborative effort from users, clinicians, designers and other professionals.

\section{References}

[1] Fliess-Douer O, Vanlandewijck YC, Van Der Woude LHV. Most Essential Wheeled Mobility Skills for Daily Life: An International Survey Among Paralympic Wheelchair Athletes With Spinal Cord Injury. Arch. Phys. Med. Rehabil. 2012;93:629-635.

[2] Morgan KA, Engsberg JR, Gray DB. Important wheelchair skills for new manual wheelchair users: health care professional and wheelchair user perspectives. Disabil. Rehabil. Assist. Technol. 2017;12:28-38.

[3] Gagnon D, Koontz A, Mulroy S, et al. Biomechanics of Sitting Pivot Transfers Among Individuals with a Spinal Cord Injury: A Review of the Current Knowledge. Top. Spinal Cord Inj. Rehabil. 2009;15:33-58.

[4] Forslund EB, Jørgensen V, Franzén E, et al. High Incidence of Falls and Fallrelated Injuries in Wheelchair Users with Spinal Cord Injury: A Prospective Study of Risk Indicators. J. Rehabil. Med. 2017;49:144-151. 
[5] Hogaboom NS, Fullerton BF, Rice LM, et al. Ultrasound Changes, Pain, and Pathology in Shoulder Tendons after Repeated Wheelchair Transfers. Bellevue, Washington; 2013 [cited 2016 Feb 9]. Available from:

http://www.resna.org/sites/default/files/legacy/conference/proceedings/2013/PD F\%20Versions/Mobility/Student\%20Scientific/Hogaboom.pdf.

[6] Gutierrez DD, Thompson L, Kemp B, et al. The Relationship of Shoulder Pain Intensity to Quality of Life, Physical Activity, and Community Participation in Persons With Paraplegia. J. Spinal Cord Med. 2007;30:251-255.

[7] Barbareschi G, Cheng T-J, Holloway C. Effect of technique and transfer board use on the performance of wheelchair transfers. Healthc. Technol. Lett. 2018;5:76-80.

[8] Barbareschi G, Holloway C. An investigation of factors affecting the performance of wheelchair transfers. Disabil. Rehabil. Assist. Technol. 2018;0:1-10.

[9] Gagnon D, Nadeau S, Noreau L, et al. Trunk and upper extremity kinematics during sitting pivot transfers performed by individuals with spinal cord injury. Clin. Biomech. 2008;23:279-290.

[10] Kankipati P, Boninger ML, Gagnon D, et al. Upper limb joint kinetics of three sitting pivot wheelchair transfer techniques in individuals with spinal cord injury. J. Spinal Cord Med. 2015;38:485-497.

[11] Koontz AM, Tsai C-Y, Hogaboom NS, et al. Transfer component skill deficit rates among Veterans who use wheelchairs. J. Rehabil. Res. Dev. 2016;53:279_294. 
[12] Tsai C-Y, Rice LA, Hoelmer C, et al. Basic Psychometric Properties of the Transfer Assessment Instrument (Version 3.0). Arch. Phys. Med. Rehabil. $2013 ; 94: 2456-2464$.

[13] Crytzer TM, Cooper R, Jerome G, et al. Identifying research needs for wheelchair transfers in the built environment. Disabil. Rehabil. Assist. Technol. $2015 ; 0: 1-7$.

[14] Sears A, Hanson VL. Representing Users in Accessibility Research. ACM Trans Access Comput. 2012;4:7:1-7:6.

[15] Iezzoni Li, McCarthy Ep, Davis Rb, et al. Mobility problems and perceptions of disability by self-respondents and proxy respondents. Med. Care. 2000;38:10511057.

[16] Bennett CL, Cen K, Steele KM, et al. An Intimate Laboratory?: Prostheses As a Tool for Experimenting with Identity and Normalcy. Proc. 2016 CHI Conf. Hum. Factors Comput. Syst. [Internet]. New York, NY, USA: ACM; 2016 [cited 2017 Sep 12].p. 1745-1756. Available from: http://doi.acm.org/10.1145/2858036.2858564.

[17] Carrington P, Hurst A, Kane SK. Wearables and Chairables: Inclusive Design of Mobile Input and Output Techniques for Power Wheelchair Users. Proc. 32Nd Annu. ACM Conf. Hum. Factors Comput. Syst. [Internet]. New York, NY, USA: ACM; 2014 [cited 2017 Sep 18].p. 3103-3112. Available from: http://doi.acm.org/10.1145/2556288.2557237. 
[18] Holloway C, Dawes H. Disrupting the world of Disability: The Next Generation of Assistive Technologies and Rehabilitation Practices. Healthc. Technol. Lett. $2016 ; 3: 254-256$.

[19] Crabtree A, Hemmings T, Rodden T, et al. Designing with care: Adapting cultural probes to inform design in sensitive settings. Ergon. Soc. Aust. 2003. p. $4-13$.

[20] Braun V, Clarke V. Using thematic analysis in psychology. Qual. Res. Psychol. 2006;3:77-101.

[21] Fereday J, Muir-Cochrane E. Demonstrating Rigor Using Thematic Analysis: A Hybrid Approach of Inductive and Deductive Coding and Theme Development. Int. J. Qual. Methods. 2006;5:80-92.

[22] Weed M. Capturing the essence of grounded theory: the importance of understanding commonalities and variants. Qual. Res. Sport Exerc. Health. 2017;9:149-156.

[23] Silverman D. Interpreting Qualitative Data: Methods for Analyzing Talk, Text and Interaction. SAGE; 2006.

[24]__ Keeler L, Kirby RL, Parker K, McLean KD, Hayden JA. Effectiveness of the Wheelchair Skills Training Program: a systematic review and meta-analysis. Disability and Rehabilitation: Assistive Technology. 2018 Apr 4;0(0):1-19.

[25] Tsai C-Y, Hogaboom NS, Boninger ML, Koontz AM. The Relationship between Independent Transfer Skills and Upper Limb Kinetics in Wheelchair Users. BioMed Research International. 2014 Aug 5;2014:e984526.. 


\section{[26]} Teeter L, Gassaway J, Taylor S, et al. Relationship of physical therapy inpatient rehabilitation interventions and patient characteristics to outcomes following spinal cord injury: The SCIRehab project. J. Spinal Cord Med. 2012;35:503-526.

[2725] Yarkony GM, Roth EJ, Meyer PRJ, et al. Rehabilitation Outcomes in Patients with Complete Thoracic Spinal Cord Injury. J. Phys. Med. 1990;69:23-27.

[2826] Worobey LA, Rigot SK, Hogaboom NS, et al. Investigating the Efficacy of Web-Based Transfer Training on Independent Wheelchair Transfers Through Randomized Controlled Trials. Arch. Phys. Med. Rehabil. [Internet]. 2017 [cited 2017 Oct 26]; Available from:

http://www.sciencedirect.com/science/article/pii/S0003999317304951.

[2927] Yamauchi S. Advanced Interdisciplinary Human Research in Assistive Technology for Elderly Persons and Persons with Disabilities. Adv. Robot. 2009;23:1455-1458.

[3028] Barbareschi G, Holloway CSM, Sprigle S. Assistive Technology Design Courses: The Mutually Beneficial Relationship between Engineering Education and the Provision of Orphan Devices. Httpwww Resna Orgnews-EventsannualMeet.-Meet.-Proceedingsannual-Conf.-Proc. [Internet]. Rehabilitation Engineering and Assistive Technology Society of North America; 2017 [cited 2017 Sep 12]. Available from: http://discovery.ucl.ac.uk/1547624/.

[3129] A JG Koontz, Crytzer TM, Cooper R. Proceedings of the Web-Based Workshop on Independent Wheelchair Transfers (IWT) [Internet]. Human Engineering 
Research Laboratories; 2012. Available from:

$<$ http://herl.pitt.edu/ab/IWT_Sept_26_Workshop\%20Proceedings(final).pdf $>$.

[3230] Goffman E. The Presentation of Self in Everyday Life. 1959; 